\title{
Bioanalysis
}

\section{Highlights from the 2014 Applied Pharmaceutical Analysis Conference}

The 10th annual Applied Pharmaceutical Analysis (APA) conference was held from 8th to 10th September in Cambridge, MA, USA. This year's APA conference focused on three different 'workshops' over the 3 days: Regulated Bioanalysis, Biotransformation, and Discovery. There was a great amount of information discussed by a variety of experts over the 3 days. This included, among other things; speakers from the US FDA discussing statute changes and guidelines, leaders from academic laboratories discussing innovation in bioanalytical tools, and industry scientists discussing current trends in the industry. The conference afforded attendees the opportunity to learn from the speakers during their sessions. In addition, there was ample opportunity for attendees and speakers both to learn from each other through informal interactions.

Keywords: APA • conference report • regulated bioanalysis • The Boston Society

The Applied Pharmaceutical Analysis (APA) conference is organized by the Boston Society, a non-profit organization founded in 2004 as a forum to facilitate education, training, discussion and scientific activities within the industry. The organization includes scientists in several areas of research and development from academia, industry and government [1]. The APA conference puts an emphasis on attendee programming interest and participation for the annual meeting. This was reiterated by the different sessions chairs requesting for the attendees for additional volunteers for the upcoming year. This report is focused on those aspects of the three workshops that pertained to bioanalysis, although several other topics were discussed in detail.

\section{Day 1}

After a brief introduction to the conference by Dr Ragu Ramanathan, the Plenary speaker for day 1, Dr Michael Skelly of the US FDA, was introduced and spoke on the State of the FDA. Dr Skelly's focus of this talk was on the changes to statutes, regulations, guidances and updates on the FDA's bioequivalence inspection program.
Dr Skelly started by discussing certain aspects of the Biologics Price Competition and Innovation Act of 2009 (part of the Patient Protection and Affordable Care Act passed in March of 2010). This act provided a path forward, not only for biosimilar approval, but also laid the groundwork for providing an additional designation of interchangeable (section $351[\mathrm{k}]$ of the Public Health Service Act). While the guidances providing specifics around biosimilar approval are still only in the draft stage, there is evidence of the EMA and FDA harmonization regarding the approval processes. In conclusion to this topic, Dr Skelly outlined the content of a biosimilar application.

Dr Skelly then dedicated time to the topic of inspections. There were two items in particular that were brought up. The first was a general 'rule of thumb' to always be polite and professional when dealing with the FDA or other regulatory inspectors. This was pertinent as there has been some feedback to the FDA that a few newer inspectors have been treating the FDA guidance documents as binding. Dr Skelly noted that before taking any action, one should ensure the inspector is
Franklin P Spriggs $*, 1$ \& Rebecca Crisino ${ }^{2}$ 'Pfizer, Inc.,1 Burtt Rd, Andover, MA 01810, USA

2Janssen R\&D, LLC, 200 Great Valley Pkwy, PA, USA

*Author for correspondence:

Tel.: +19782471003

Fax: +1 8454743613

franklin.spriggs@pfizer.com 
actually treating the guidance as binding and not simply asking questions regarding the work being done, and how it does or does not follow the guidance. If after this, you feel the inspector is in fact treating a guidance as a binding document, Dr Skelly suggested you contact that inspector's supervisor in the center office or the office that issued the guidance document. Also related to inspections was the Generic Drug User Fee Act (GDUFA) mandate that the FDA consider inspections by other regulatory agencies and encourage cooperation. To this end, it was suggested that when the FDA conducts an inspection at your facility, and you have been inspected by a partner agency (such as the EMA), you can inform the FDA of this inspection and/or provide the inspection report from that partner agency.

Dr Skelly concluded his talk by outlining some items that could hinder an inspection, items that neither hurt nor help an inspection, and those items that help during a regulatory inspection. Those items that hinder inspections include: delaying or refusing an inspection; altering records to confound inspections; refusing to correct objectionable conditions; cut-andpaste errors; and incomplete reports. Items that neither help nor hinder are: errors in grammar/spelling/word usage; excessive signatures; and claims of compliance with inappropriate regulations. Finally, those things that can help an inspection go smoothly include: legible entries; indexed, hyperlinked, and searchable reports; justification for non-standard operations; and sharing of audit reports from other regulatory agencies.

Following the Plenary session there was a regulated bioanalysis session focused on guidance in the industry. The session was chaired by Dr Farhad Sayyarpour (Eurofins) and Eric Woolf (Merck).

Several broad topics, as well as some more specific information, were discussed in this first session. One topic was regarding the determination of whether a study should or should not be conducted in a GLP manner. This question should be answered during the planning stages of a study, and depends on what is the intended use of the data. Regardless of how the data will support a program; does the requested data match the intended use of this data?

Once it has been determined that a study should be conducted under the GLP regulations, all the bioanalytical method validation (BMV) defined parameters should be evaluated. It was again stressed during this first session the differences between regulations, guidelines and white papers and how they differ from the viewpoint of the FDA.

Incurred sample reanalysis (ISR) was discussed at some length by multiple speakers as it is a standalone section within the 2013 Draft Bioanalytical Method
Validation FDA guidance [2], and was also discussed at Crystal City V. The proposed number of samples to be run was set at $7 \%$ and it was noted that there is a strong preference to align requirements globally. While the percentage of samples to enter ISR was discussed, one point that was conspicuously missing was a minimum number of samples required for smaller studies. One speaker also stressed that ISR can also be beneficial beyond just demonstrating reproducibility of the assay, such as being useful in issue resolution as part of investigations.

A second parameter that was discussed in some detail was how to target and set an analytical assay range that best suits the needs of a specific program. This can be difficult depending on the stage of the compound. One complication that can arise is when a project is in the earlier stages and the anticipated range is not known, or, as the dosing gets higher the samples will fall out of the range of quantitation. When this occurs, there are several options available. The first and likely most complicated would be to develop and validate two different ranges for the assay. Perhaps a more common approach is to test diluting the sample so the concentration falls within the already validated quantitative range. When doing this, it was suggested that a sample be prepared with reference material and the highest dilution required for sample analysis be tested.

Another point to note was related to matrices, specifically use of rare and/or difficult to obtain matrices. When conducting bioanalysis for a study, it has been the rule that the matrix of the samples should be the same type of matrix. On occasion this is not possible, prohibitively expensive, or not morally acceptable.

Following information on bioanalytical guidance documents, a session with a focus on antibody drug conjugates (ADCs) was conducted. The Session was chaired by Dr Johanna Mora (Bristol-Myers Squibb) and Dr Stephanie Farmer (Frontage Labs).

The session started off by providing a background of ADCs, then acknowledging the rapid growth of ADCs in the last several years as well as the growing market potential of ADCs [3,4]. As part of the background of ADCs, examples regarding the challenges a bioanalytical laboratory can expect to encounter when developing an ADC therapeutic were detailed. These included both management challenges due to capacity and safety considerations, as well as laboratory and scientific challenges specific to ADCs.

One area of focus for the speakers was assessment of stability in different species and matrices. In one case study a strategy was outlined on how to assess ADC stability in whole blood and plasma. In this case study there was an observed loss of stability of the linkerpayload ex vivo that needed to be resolved in order to 
obtain accurate results for on-going study support. Through thorough testing and a systematic approach it was determined that reducing the $\mathrm{pH}$ in the sample collection tubes (acid citrate dextrose [pH 5]) was able to slow the release of the linker/payload species, possibly due to free thiols present in the plasma reacting with free drug linker.

In a second case study, there was compound that demonstrated excellent stability in humans and nonhuman primates (NHP); however, the stability was very different in rodents. Through HPLC analysis, it was determined this was due to drug payload modifications that happened very quickly in the rodent (within minutes). These same modifications occurred in the human and NHP took days for these same modifications. Through time and with well-designed experiments a specific reagent was identified that was able to recognize all the active forms of the drug modifications. A follow-up that was conducted after determining the best assay reagent to use was able to determine that the likely cause of the drug payload modifications was due to the amount of esterase in the different species evaluated.

\section{Day 2}

Day 2 started with a session on the development and quantitation of proteins and peptide therapeutics chaired by Dr Natasha Penner (Biogen Idec) and Dr Scott Fountain (Pfizer).

The session was started with a talk on profiling and imaging of thin tissue sections. After a brief introduction on different ambient surface sampling methods, alternative methods of tissue profiling were presented. These included methodologies that have previously been published by the presenting author and will not be discussed in great detail here [5-11]. The following talks focused on methodologies developed for the quantitation of biomarkers in support of clinical programs.

One method presented an immunoaffinity LC-MS/MS approach used to provide data for pharmacokinetic and pharmacodynamics models. This talk outlined challenges associated with the project, including differences between target expression in different species and an apparent disconnect between in vitro potency and in vivo efficacy. After outlining the different immunoaffinity workflows and the advantages/disadvantages, the performance of the chosen method was presented, as well as a proof-of-concept on the method showing expected target engagement from an in vivo model.

Another approach utilized an ultra-centrifugation (UC) method to discover and conduct ADME profiling of a novel long-acting potent peptidic GLP-2 agonist. This talk outlined the UC method used to screen many different peptides in multiple species and assess stability of lead compounds when required. Using this approach and making it possible to execute it for a large number of samples in a short period of time allowed the discovery of compounds that demonstrated desirable ADME characteristics by screening for compounds with enhanced half-life and exposure while retaining adequate potency.

The next session was focused on biomarkers in drug development and was chaired by Mr Rick Steenwyk (Pfizer) and Dr Yongjun Xue (Celgene).

This session began with how biomarkers are used to establish specific clinical parameters, such as proof-ofconcept, patient stratification, and so on. As the session progressed, several different methodologies were discussed on how to go about measuring different clinical biomarkers. One such methodology presented spoke to the emerging role of LC-MS/MS for clinical protein biomarkers. Regarding the use of LC-MS/MS, the process was outlined and included some guidelines for when protein digestion would be needed and when a protein can be evaluated without such a step (i.e., proteins $\leq 5 \mathrm{kDa}$ ). Additional to these guidelines there were key benefits and issues that were recognized and presented. A key benefit to LC-MS/MS is the specificity and usability of an internal standard, where some examples of the issues brought forward is the need to produce a recombinant internal standard and insufficient sensitivity for low abundance biomarkers.

The other methodology presented in detail was ligand-binding assays (LBAs), specifically multiplexing of LBAs. Two platforms capable of multiplexing were used as examples, Luminex ${ }^{\circledR}$ and Meso-Scale Discovery ${ }^{\circledR}$. The multiplexing of LBAs has unique challenges, including using a single minimum required dilution, crossreactivity, and matrix interference. While these parameters are also executed with a singleplex assay, it becomes more difficult when evaluating for multiple analytes in a single well/bead.

The final session of day 2 related to bioanalysis was on the topic of limits of sensitivity and overcoming matrix interference in LBA and MS assays, chaired by Dr Binodh DeSilva (Bristol-Myers Squibb) and Dr Fumin Li (PPDI).

To start the session a major challenge associated with measuring biomarkers in biofluids was highlighted, that being the wide dynamic range, sensitivity and specificity requirements [12]. This challenge was presented as a major impediment for LC-MS/MSbased assays as the sensitivity for proteins is generally lower than that of LBAs. It was proposed that sensitivity could be dramatically enhanced by improving the LC-MS/MS interface to improve ionization, ion 
sampling, and ion transmission efficiencies. To this point, several innovative methods were presented, specifically a new strategy termed PRISM-SRM MS [13] and the development of a penta quadrapole MS instrument to further improve sensitivity.

The session continued with an introduction and update of an American Association of Pharmaceutical Sciences working group: the Emerging Technology Discussion Group. After outlining the key activities of the sensitivity and assay interference teams within this discussion group, the technologies under evaluation were presented. For sensitivity improvement the technologies under consideration were immunePCR (Chimera Biotek), SMC/Errena (Singulex) and SiMoa (Quanterix). The assay interference team, it was explained, is collaborating with several manufacturers to develop and execute a comparison between four different platforms in order to objectively evaluate assay interference improvements. The platforms under evaluation are: SMD/Errena (Singulex), SiMoa (Quanterix), Ig_Plex (SQI Diagnostics), and Maverick (Genalyte).

The final presentation of the session was on the Bioanalysis of PEGylated proteins by quantitative NMR. This presentation outlines various issues with standard approaches to quantitating PEGylated proteins. These issues included: non-specific reagents, radiolabeling problems, MALDI-MS issues with degradation, and selective ionization, among others. The concept of using NMR for quantitation is not new; however, it is novel for quantitation of high molecular weight PEG species. There are several considerations identified during the process of development and validation of the methodology that need to be taken into account, such as, determining what the validation criteria should be (followed chromatographic guidance), identification of best reference material (Fab-PEG in this case), what are the regulatory issues, how can LBA and NMR data be compared.

\section{Day 3}

Day 3 brought the focus back to bioanalysis with a session titled Bioanalytical Strategies for Novel Therapies and Emerging Analytical Methods, chaired by Dr Jing Tao Wu (Takeda) and Dr Montserrat Carrasco-Triguero (Genentech).

This session started with a presentation that highlighted the FDA's Question Based Review concept and provided some case studies that highlighted the regulatory perspective on bioanalysis. During the course of this presentation there were some key pharmacology questions, such as: what is the systemic exposure-response relationship of the drug for safety/efficacy? What intrinsic and extrinsic factors influence exposure and/or response and what is the impact of any differences in exposure on efficacy or safety responses? There were also key bioanalytical questions presented, such as: is the method reproducible? What methods were used to ID/quantitate the parent drug and metabolites? What metabolites have been selected for analysis and why? The speaker continued with some case studies that all had a common theme. This theme was to highlight the advantages of early communication with the FDA during the course of a drug-development problem. This early communication may help both the drug company as well as the FDA to understand the potential pitfalls to certain studies and bioanalytical methodologies before excess time and money has been spent answering questions that may have been the wrong questions to ask.

The next two speakers focused on the bioanalysis of ADCs. The first of these talks discussed the complexity of ADCs and how the conjugation chemistry, associated heterogeneity, and ADC biotransformation introduce unique challenges in bioanalysis. The complexity of these programs in compounded by the fact that drug to antibody ratio (DAR) can change in plasma over time [14]. The speaker concluded, after presenting information on recent clinical data, that there are limited agency guidelines for choosing analytes for safety and efficacy.

The final speaker of the session outlined what analytes need to be measured when running an ADC program. As part of this talk, the most commonly assessed analytes were presented from a recent position paper. These included: conjugated antibody, total antibody, ADC, unconjugated drug and anti-ADC antibody [15]. Additional information was then presented on the differences between unconjugated drug versus conjugated drug as measured by LC-MS/MS. This distinction is critical as unconjugated drug is an important component of safety, whereas conjugated drug is essential to efficacy, and possibly safety as well.

As discussed in the first talk of this session, the ultimate goal is to answer key pharmacology questions is to determine what the exposure/response relationship is then correlate that to efficacy and safety signals. Differences in how this data was evaluated were presented in the form of case studies. One such case study showed how assay results (exposure) differ with the DAR value. Another asked the question, is there a need to characterize domain specificity of anti-ADC antibodies? (Maybe, depends on the program needs). Finally, bioanalytical method changes throughout the life of an ADC program can, and often do change. These changes can pose challenges if the analyte being measured is not the same (i.e., use of a generic IgG vs specific assays) and may require assay redesign. 
In conclusion to this session, it was noted that while it is difficult to know which of these analytes contributes to efficacy and which contributes to the safety profile, it is important to compile a complete and accurate description of ADC exposure through the bioanalysis of a defined set of analytes.

\section{Conclusion}

The 2014 APA conference was well-attended and allowed for easy cross-discipline interactions. These interactions, as well as the pre-conference workshops and excellent presentations given throughout the main conference, presented a wonderful forum for learning not only new topics, but also expanding existing knowledge.

The conference continues to focus on member focused programming in regulated bioanalysis, biotransformation and discovery sciences in the pharmaceutical and

\section{References}

\section{Society TB}

www.bostonsociety.org/about-us.html

2 US FDA. Services USDOHAH, Research CFDEA, Medicine CFV, Administration FAD. In: Draft Guidance For Industry: Bioanalytical Method Validation (2013).

www.fda.gov/downloads/drugs/

guidancecomplianceregulatoryinformation/guidances/ ucm368107.pdf

3 Analysis R. Antibody Drug Conjugates Market, 2013-2023. www.rootsanalysis.com/reports/view_document/antibodydrug-conjugates-market-2013-2023/21.html

4 Development DDa. ADCs Represent a Coming Wave for Drug Developers: Drug Discovery and Development. www.dddmag.com/news/2014/01/adcs-represent-comingwave-drug-developers

5 Kertesz V, Van Berkel GJ. Liquid microjunction surface sampling coupled with high-pressure liquid chromatography-electrospray ionization-mass spectrometry for analysis of drugs and metabolites in whole-body thin tissue sections. Anal. Chem. 82(14), 5917-5921 (2010).

6 Kertesz V, Van Berkel GJ. Automated liquid microjunction surface sampling-HPLC-MS/MS analysis of drugs and metabolites in whole-body thin tissue sections. Bioanalysis 5(7), 819-826 (2013).

7 Kertesz V, Van Berkel GJ, Vavrek M, Koeplinger KA, Schneider BB, Covey TR. Comparison of drug distribution images from whole-body thin tissue sections obtained using desorption electrospray ionization tandem mass spectrometry and autoradiography. Anal. Chem. 80 (13), 5168-5177 (2008). biotechnology industries. As mentioned earlier, there were a large number of topics presented; however, only those areas specifically relevant to bioanalytical sciences have been discussed here. The next APA conference will be held on 14-16th September 2015 in Cambridge, MA, USA.

\section{Financial \& competing interests disclosure}

The authors are employees of Pfizer, Inc and Janssen Research and Development, LLC, a division of Johnson \& Johnson, Inc., and own company stock. The authors have no other relevant affiliations or financial involvement with any organization or entity with a financial interest in or financial conflict with the subject matter or materials discussed in the manuscript apart from those disclosed.

No writing assistance was utilized in the production of this manuscript.

8 Van Berkel GJ, Kertesz V. Application of a liquid extraction based sealing surface sampling probe for mass spectrometric analysis of dried blood spots and mouse whole-body thin tissue sections. Anal. Chem. 81(21), 9146-9152 (2009).

9 Van Berkel GJ, Kertesz V. Continuous-flow liquid microjunction surface sampling probe connected on-line with high-performance liquid chromatography/mass spectrometry for spatially resolved analysis of small molecules and proteins. Rapid Commun. Mass Spectrom. 27(12), 1329-1334 (2013).

10 Van Berkel GJ, Kertesz V, King RC. High-throughput mode liquid microjunction surface sampling probe. Anal. Chem. 81(16), 7096-7101 (2009).

11 Van Berkel GJ, Kertesz V, Koeplinger KA, Vavrek M, Kong AN. Liquid microjunction surface sampling probe electrospray mass spectrometry for detection of drugs and metabolites in thin tissue sections. J. Mass Spectrom. 43(4), 500-508 (2008).

12 Anderson NL, Anderson NG. The human plasma proteome: history, character, and diagnostic prospects. Mol. Cell. Proteomics 1(11), 845-867 (2002).

13 Shi T, Fillmore TL, Sun X et al. Antibody-free, targeted mass-spectrometric approach for quantification of proteins at low picogram per milliliter levels in human plasma/serum. Proc. Natl Acad. Sci. USA 109(38), 15395-15400 (2012).

$14 \mathrm{Xu} \mathrm{K}, \mathrm{Liu} \mathrm{L}, \mathrm{Saad} \mathrm{OM}$ et al. Characterization of intact antibody-drug conjugates from plasma/serum in vivo by affinity capture capillary liquid chromatography-mass spectrometry. Anal. Biochem. 412(1), 56-66 (2011).

15 Gorovits B, Alley SC, Bilic S et al. Bioanalysis of antibodydrug conjugates: American Association of Pharmaceutical Scientists Antibody-Drug Conjugate Working Group position paper. Bioanalysis 5(9), 997-1006 (2013). 